\title{
Adaptive Strategic Variations in Human Cognition Across the Life Span
}

\author{
Thomas Hinault \\ CNRS \& Aix-Marseille Université \\ 3 Place Victor Hugo, 13331 Marseille France \\ Tel: (+33)4.13.55.09.68 Email: thomas.hinault@univ-amu.fr \\ Patrick Lemaire (Corresponding author) \\ CNRS \& Aix-Marseille Université \& CNRS \\ 3 Place Victor Hugo, 13331 Marseille France \\ Tel: (+33)4.13.55.09.85 Email: patrick.lemaire@univ-amu.fr
}

\begin{abstract}
Received: January 8, 2016 Accepted: January 17, 2016 Published: January 31, 2016
doi:10.5296/jet.v3i1.8967

URL: http://dx.doi.org/10.5296/jet.v3i1.8967
\end{abstract}

\begin{abstract}
This paper provides an overview of how a strategy perspective fruitfully contributes to our understanding of psychological adaptation in problem-solving tasks, as well as how strategic adaptation develops across lifespan. Indeed, people do not use a single strategy to solve various problems, nor do their strategies remain the same across their lifespan. Problem-solving performance is determined by efficient strategy selection and execution, and strategy effectiveness is modulated by characteristics of problems, strategies, situations, and participants. Multiple strategy use help participants to obtain better performance through strategic adaptations. Strategic adaptations can be defined as participants' calibrations of how they accomplish cognitive tasks as a function of different task parameters. Moreover, this review consider how strategic adaptation mechanisms are implemented during childhood, as well as aging effects on the ability to select and execute strategies adaptively given environmental constraints. Third, the role of working memory capacity and executive processes in strategy use and in age-related changes in strategy adaptativeness are discussed. This review illustrates developmental changes of strategic adaptation during childhood and adulthood with findings from a variety of cognitive domains, including decision making and arithmetic problem solving.
\end{abstract}

Keywords: Strategies, Adaptation, Cognitive Development, Aging, Children 


\section{Introduction}

Decades of research on human cognition have established that people use a wide variety of strategies to accomplish cognitive tasks (e.g., Siegler, 2007). A strategy is "a procedure or a set of procedures for achieving a higher level goal or task" (Lemaire \& Reder, 1999, p. 365). Multiple-strategy use helps participants to obtain better performance through strategic adaptations. Strategic adaptations can be defined as participants' calibrations of how they accomplish cognitive tasks as a function of different task parameters. Strategic adaptations can be studied by investigating how participants calibrate their strategy use and strategy execution to task environments while they accomplish cognitive tasks. This article first discuss how adaptive participants are when they accomplish cognitive tasks, as seen in how they change strategies as a function to main task parameters. Next, data on how calibrations of strategy use and strategy execution to task constraints change with participants' age during childhood as well as during adulthood are reviewed. Moreover, the role of executive functions in strategic adaptations and age-related changes therein is discussed. Executive functions refer to a set of top-down processes needed when automatic processing is not possible, or would be ill-advised (see Diamond, 2013, for a review). All in all, the data reviewed here on age-related changes in strategy adaptiveness during lifespan helps us understand what factors influence strategic adaptations, and characterize what cognitive processes qualify for adaptivity.

\section{Strategic adaptations in human cognition}

It has been long known that participants do not accomplish cognitive tasks with a single strategy (see Siegler, 2007, for a review). Also, participants select strategies on a problem-by-problem basis. That is, they do not decide to solve a first series of problems with one strategy, a second series with another strategy, a third with yet another strategy, and so on. Quite the contrary, both children and adults of all ages select strategies on each problem. They also do not select strategies randomly. Usually, participants' try their best to use the best strategy on each item. Decades of research in cognitive psychology have established that strategy selection and strategy execution are influenced by participants, items, strategies, and situation characteristics. This can be illustrated with a few examples.

In the domain of simple arithmetic problem solving, where people have to determine what is the correct answer to problems like $3 \times 4$ or $48+59$, sometimes they use counting strategies (adding 3 four times), and sometimes they retrieve the correct solution (12) directly from memory. People tend to use retrieval strategy more often than counting on problems with small operands, like $3 \times 4$, and counting more often on larger problems (e.g., 7x8; LeFevre et al., 1996). Also, participants are faster when they execute counting strategies on small relative to larger problems. The difference in performance between smaller and larger problems is bigger when participants use counting than when they use retrieval. Similarly, in episodic memory, both children and adult participants can learn lists of words with diverse strategies like mental image (i.e., mentally visualizing a word during encoding) or rote-repetition (i.e., continuously repeating words). Mental image strategy is usually more efficient than rote-repetition, especially on concrete words (e.g., Dirkx \& Craik, 1992; 
McGilly \& Siegler, 1989).

As a final example, when participants accomplish reasoning tasks, they also use different strategies. Strategies differ in relative performance, and differences in strategy performance are modulated by the type of items on which people reason. To illustrate, in a verbal reasoning task, where participants were shown sentences and pictures on a computer screen, participants had to decide whether sentences describe pictures. Participants' performance differed if participants used a verbal or a visual imagery strategy (Reichle et al., 2000). That is, participants were presented pictures showing, for example, a triangle above a square. Under the picture, participants could read sentences like "It is true that a triangle is not under the square". Sentences varied in whether they were positive or negative, and whether they were active or passive. Even if participants were faster with visual imagery strategy (i.e., trying to make a mental image with the sentence before comparing this image with the proposed image) than with a verbal strategy (i.e., not making a visual image with the sentence before comparing the content of the sentence with that of the picture), this difference varied as a function of how the sentence was formulated. For example, the difference between visual imagery strategy and verbal strategy was larger for true, negative sentences ("it is true that the square is not above the triangle") than for true positive sentences ("It is true that the triangle is above the square").

Another crucial factor that influences participants' strategy selection and strategy execution is situation characteristics (e.g., speed/accuracy emphasis). For example, Campbell and Austin (2002) asked participants to solve simple arithmetic problems like $2+3$ or $8+7$ under a fast deadline (i.e., respond before $750 \mathrm{~ms}$ ) or a slower deadline (i.e., respond after $2500 \mathrm{~ms}$ ). Direct retrieval in memory increased under fast deadline condition, especially for larger problems. In other words, to cope with task demands of responding quickly, participants increased their use of the fastest strategy.

Also, participants adjust strategy use to their skills at executing available strategies. For example, Gandini and her collaborators (2008a, b; 2009) conducted a series of experiments in which they asked participants to estimate numerosity of collections including between 30-200 dots, as quickly as possible. The authors evaluated how fast and precise each participant was when forced to use two most commonly used strategies: benchmark (i.e., participants visually estimated the number of dots) or anchor (i.e., participants approximately counted groups of dots, and then added these numbers) strategies. Participants tended to use the benchmark strategy on collections of dots for which they could be fastest and most accurate, and the anchor strategy on all the other collections. In other words, each individual was selecting the strategy that was most efficient for him or her on each item.

In sum, previous research in cognitive psychology has found that participants do not rigidly use a single strategy to accomplish cognitive tasks, but use multiple strategies. The usefulness of multiple-strategy use is that it enables participants to optimize their performance, because some strategies are more efficient on some problems, and other strategies on other problems. Participants try to obtain best performance through strategic adaptations. Strategic adaptations consist in fine calibrations of which strategy is used on a given problem and of 
how each strategy is executed to different task parameters like problems and situations features. Strategic calibrations also involve skills at adjusting strategy use to our own ability to execute each strategy efficiently. Of crucial interests is how such calibrations change with participants' age both during childhood and during aging.

\section{Age-related changes in strategic adaptations during childhood}

Few studies have been conducted to determine how strategic adaptations are implemented and evolve throughout childhood. With age, children use the most efficient strategy more often and show higher correlation between strategy use and task parameters (Barrouillet \& Lépine, 2005; Imbo \& Vandierendonck, 2007; LeFevre et al., 1996; Lemaire \& Siegler, 1995; Otto et al., 2006; Quaiser-Pohl et al., 2010; see Siegler, 1996, for an overview). For example, in arithmetic, repeated practice of problems solving lead to memorization of problem-response associations. Then, compared to counting, problems can be solved faster and more efficiently by direct retrieval of arithmetic facts from long-term memory. Indeed, proportion of problems solved with retrieval strategy increases throughout childhood, starting from $22 \%$ in five-year-olds to reach $80 \%$ in adults (Imbo \& Vandierendonck, 2007; LeFevre et al., 1996). Also, differences in performance between small and larger problems decrease between 9-year-old and 11 year-old. These differences are no longer different from those in adults after 11 year-old (De Brauwer et al., 2006). This result implies that children have developed an arithmetic facts network similar to adults' network. This arithmetic facts network enables children to use efficiently retrieval strategy when available and counting strategies for other problems (see also Campbell \& Graham, 1985; Koshmider \& Ashcraft, 1991; Lemaire et al., 1994). Likewise, in memory, increased performance from five to eight year-old to recall list of numbers is closely related to increased use of repeated rehearsal and decreased use of single rehearsal or no rehearsal strategies (Fabricius \& Cavalier, 1989; McGilly \& Siegler, 1989). Older children tended to use more often repeated rehearsal strategy to recall list of numbers when they were unable to recall the list correctly on the previous trial, thus adjusting strategy use to improve performance.

Strategic adaptations are also modulated by situations. For example, in an approximate quantification task, Gandini et al. (2010) asked children to estimate numerosities of collections of dots. Dots were displayed either randomly or canonically (e.g., three dots displayed like a triangle, four like a square). Children produced better estimates on canonical than on random displays, and that these canonical-random differences decreased between 10 and 12 year-old.

Age-related changes in strategic adaptations also differ as a function of individuals' skills. Interestingly, Barrouillet and Lépine (2005) revealed that children with high working memory capacity use retrieval strategy more often than children with low working memory capacity. This difference between groups was more important in large problems. Indeed, children with high working memory capacity are able to use counting strategies more efficiently on larger problems, and are able to use retrieval strategies on more problems than children with low working memory capacity. In addition to working memory capacity, executive functions were also found to play an important role in the development of strategic 
adaptations during childhood. Results revealed that children with the highest scores in tests measuring executive functions also showed the best strategy selection performance (e.g., Lemaire \& Lecacheur, 2011). Moreover, efficiency of executive functions contributed to improvement of children's strategic adaptations between 8 and 12 year-old.

\section{Age-related changes in strategic adaptations during aging}

In many cognitive domains and tasks, aging is mostly, though not always, related with decline strategic adaptations relative to young adults. Indeed, older adults tend to favor the less demanding strategies, even if they are not the best (e.g., Duverne et al., 2003, 2007; Duverne \& Lemaire, 2004, 2005; Gandini et al., 2009; Green et al., 2007; Hodzik \& Lemaire, 2011; Lemaire et al., 2004; Fein et al., 2007; Mata et al., 2007, 2010; Mata \& Nunes, 2010). As an example, in estimation calculation tasks, participants can estimate products of two-digit multiplication problems with several strategies. Participants were asked to use a rounding-down strategy (i.e., rounding both operands down to the nearest decades, like doing $40 \times 60$ to estimate $43 \times 68$ ), or a rounding-up strategy (i.e., rounding both operands up to the nearest decades, like doing 50x70 to estimate 43x68; Hodzik \& Lemaire, 2011; Lemaire et al., 2004). The rounding-down strategy is a better strategy (i.e., that yields the closest estimate to the exact product) to estimate products when unit-digits are smaller than five, while the rounding-up strategy is better to estimate problem when unit-digits are larger than five. Hodzik and Lemaire observed decline strategic adaptations in older adults, as they used less often than young adults the rounding-down strategy when unit digits of multiplication problems were smaller than five, and the rounding-up strategy when unit digits were larger than five. Furthermore, age-related differences in use of the best strategies was larger on hard problems (e.g., 47x63; 24x76) than on easy problems (e.g., 43x54; 37x89). Thus, despite longer experience of calculation, strategy selection became less adaptive with age.

Age-related decline in strategic adaptations is modulated by situations, as aging effects can be reduced or increased in specific situations. In memory, older adults were found to use retrieval strategy less often to retrieve pairs of words, leading to poorer performance compared to young adults (Bailey et al., 2009; Dunlosky \& Hertzog, 2001; Frank et al., 2013; Naveh-Benjamin et al., 2005; Touron \& Hertzog, 2004, 2009). However, when information was given about strategies (e.g., retrieval strategy is more efficient that rote-repetition strategy), age difference in strategy use was reduced (see also Froger et al., 2012). In addition to information about strategies, Froger et al. (2012) also studied time to encode pairs of words. When study time was not limited, older adults spent more time and adjusted their strategy more efficiently to characteristics of words (e.g., more frequent use of mental-image when associations between words are stronger compare to weaker associations). Age-related differences in memory performance were thus reduced, compared to when study time was limited to six seconds. Conversely, time pressure leads to larger age-related differences in recall performance than no time pressure (e.g., Earles et al., 2004).

Moreover, important individual differences can be found regarding how strategic adaptation evolves with age. Indeed, several life factors (e.g., education, lifestyle) enable some older adults to benefit from a cognitive reserve. Cognitive reserve is the ability to cope with 
age-related changes in the brain with larger cerebral recruitment to preserve cognitive functions (Stern, 2009). Older adults with high cognitive reserve were found to show higher strategic adaptations than other older adults, as they selected the best strategy on each problem more often and executed strategies more efficiently (Barulli et al., 2013). Executive functions also modulate preservation or alteration of strategic adaptations during cognitive aging. Indeed, it was observed that older adults with the highest scores in measures of executive functions executed strategies more efficiently and selected the better strategy on each problem more often than older adults with lower scores (e.g. Hodzik \& Lemaire, 2011).

\section{Conclusion}

A strategy perspective is fruitful to better understand processes involved during adaptation as a function of task parameters. Indeed, strategy performance was found to be modulated by characteristics of problems, strategies, situations, and participants. Even if some strategies are more efficient than others, these strategies can be better suited to some environment or problems, and less appropriate to others. Moreover, situational constraints can modulate strategy use. Furthermore, in addition to individual differences in strategy adaptativeness, strategic adaption processes evolve across lifespan. Recent studies suggest that most of the changes that occur with age in strategic adaptation processes are related to demands in working memory, and efficiency of cognitive control mechanisms.

Several cognitive processes underlie changes in strategy adaptations from childhood to adulthood, as well as between young and older adults. Strategic processing during childhood is closely related to working memory capacity. Indeed, memory span correlates more highly to memory strategy in nine year-old than in eight year-old children (e.g., Barrouillet \& Lépine, 2005). Thus, strategy adaptations become more efficient with age together with working memory resources, as more demanding strategies or problems are processed more efficiently. Also, executive functions allow to dynamically adjust strategy performance to task parameters. Age-related improvement in executive processes contributes substantially to improved strategic adaptations between 8 and 12 year-old (Lemaire \& Lecacheur, 2011). Higher control enables to more efficiently disengage from less appropriate strategies to activate a set of more efficient strategies and to flexibly switch strategies as a function of problem or situation.

Age-related decline in working memory and executive functioning also underlie changes in strategy adaptations. Indeed, 39\% of the age-related variance in percent use of the best strategy was accounted for by measures of executive functions (Hodzik \& Lemaire, 2011). Moreover, executive functions mediated use of memory strategies, as older adults with highest level of executive functions used more often and more efficiently internal memory strategies (Bouazzaoui et al., 2010). This suggests that the capacity to inhibit a poorer strategy, to maintain a set a strategy in working memory, or to flexibly switch strategies from one trial to the next decline with age and is responsible, at least in part, of less adaptive strategy processing in older adults. At a more general level, throughout lifespan, psychological adaptation seems to be efficient as long as processing resources match demands of the environment. 


\section{Acknowledgement}

The research is financed in part by the CNRS (French NSF) and by a grant from the Agence Nationale de la Recherche (Grant \# BLAN-1912-01).

\section{References}

Bailey, H., Dunlosky, J., \& Hertzog, C. (2009). Does differential strategy use account for age-related deficits in working-memory performance? Psychology and Aging, 24(1), 82-92. http://doi.org/10.1037/a0014078

Barrouillet, P., \& Lépine, R. (2005). Working memory and children's use of retrieval to solve addition problems. Journal of Experimental Child Psychology, 91(3), 183-204. http://doi.org/10.1016/j.jecp.2005.03.002

Barulli, D. J., Rakitin, B. C., Lemaire, P., \& Stern, Y. (2013). The Influence of Cognitive Reserve on Strategy Selection in Normal Aging. Journal of the International Neuropsychological Society, 19(07), 841-844. http://doi.org/10.1017/S1355617713000593

Bouazzaoui, B., Isingrini, M., Fay, S., Angel, L., Vanneste, S., Clarys, D., \& Taconnat, L. (2010). Aging and self-reported internal and external memory strategy uses: The role of executive functioning. Acta Psychologica, 135(1), 59-66. http://doi.org/10.1016/j.actpsy. 2010.05.007

Campbell, J. I., \& Graham, D. J. (1985). Mental multiplication skill: Structure, process, and acquisition. Canadian Journal of Psychology/Revue Canadienne de Psychologie, 39(2), 338-366. http://doi.org/10.1037/h0080065

Campbell, J. I., \& Austin, S. (2002). Effects of response time deadlines on adults' strategy choices for simple addition. Memory \& Cognition, 30(6), 988-994. http://doi.org/10.3758/ BF03195782

De Brauwer, J., \& Fias, W. (2009). A longitudinal study of children's performance on simple multiplication and division problems. Developmental Psychology, 45(5), 1480-1496. http://doi.org/10.1037/a0015465

Diamond, A. (2013). Executive Functions. Annual Review of Psychology, 64(1), 135-168. http://doi.org/10.1146/annurev-psych-113011-143750

Dirkx, E., \& Craik, F. I. (1992). Age-related differences in memory as a function of imagery processing. Psychology and Aging, 7(3), 352-358. http://doi.org/10.1037/0882-7974.7.3.352

Dunlosky, J., \& Hertzog, C. (2001). Measuring strategy production during associative learning: The relative utility of concurrent versus retrospective reports. Memory \& Cognition, 29(2), 247-253. http://doi.org/10.3758/BF03194918

Duverne, S., Lemaire, P., \& Michel, B. F. (2003). Alzheimer's disease disrupts arithmetic fact retrieval processes but not arithmetic strategy selection. Brain and Cognition, 52(3), 302-318. http://doi.org/10.1016/S0278-2626(03)00168-4 
Duverne, S., \& Lemaire, P. (2004). Age-related differences in arithmetic problem-verification strategies. The Journals of Gerontology Series B: Psychological Sciences and Social Sciences, 59(3), 135-142. http://doi.org/10.1093/geronb/59.3.P135

Duverne, S., \& Lemaire, P. (2005). Arithmetic split effects reflect strategy selection: an adult age comparative study in addition comparison and verification tasks. Canadian Journal of Experimental Psychology/Revue Canadienne de Psychologie Expérimentale, 59(4), 262. http://doi.org/10.1037/h0087479

Duverne, S., Lemaire, P., \& Vandierendonck, A. (2007). Do working-memory executive components mediate the effects of age on strategy selection or on strategy execution? Insights from arithmetic problem solving. Psychological Research, 72(1), 27-38. http://doi.org/10. 1007/s00426-006-0071-5

Earles, J. L., Kersten, A. W., Mas, B. B., \& Miccio, D. M. (2004). Aging and memory for self-performed tasks: Effects of task difficulty and time pressure. The Journals of Gerontology Series B: Psychological Sciences and Social Sciences, 59(6), 285-293. http://doi.org/10.1093/geronb/59.6.P285

Fabricius, W, V., \& Cavalier, L. (1989). The Role of Causal Theories about Memory in Young Children's Memory Strategy Choice. Child Development, 60(2), 298-308. http://doi.org/10.2307/1130977

Fein, G., McGillivray, S., \& Finn, P. (2007). Older adults make less advantageous decisions than younger adults: cognitive and psychological correlates. Journal of the International Neuropsychology Society, 13, 480-489. http://doi.org/10.1017/S135561770707052X

Frank, D. J., Touron, D. R., \& Hertzog, C. (2013). Age differences in strategy shift: Retrieval avoidance or general shift reluctance? Psychology and Aging, 28(3), 778-788. http://doi.org/10.1037/a0030473

Froger, C., Bouazzaoui, B., Isingrini, M., \& Taconnat, L. (2012). Study time allocation deficit of older adults: The role of environmental support at encoding? Psychology and Aging, 27(3), 577-588. http://doi.org/10.1037/a0026358

Gandini, D., Lemaire, P., \& Dufau, S. (2008a). Older and younger adults' strategies in approximate quantification. Acta Psychologica, 129(1), 175-189. http://doi.org/10.1016/ j.actpsy.2008.05.009

Gandini, D., Lemaire, P., Anton, J.-L., \& Nazarian, B. (2008b). Neural correlates of approximate quantification strategies in young and older adults: An fMRI study. Brain Research, 1246, 144-157. http://doi.org/10.1016/j.brainres.2008.09.096

Gandini, D., Lemaire, P., \& Michel, B. F. (2009). Approximate quantification in young, healthy older adults', and Alzheimer patients. Brain and Cognition, 70(1), 53-61. http://doi.org/10.1016/j.bandc.2008.12.004

Gandini, D., Ardiale, E., \& Lemaire, P. (2010). Children's Strategies in Approximate Quantification. Current Psychology Letters. Behaviour, Brain \& Cognition, 26(1). 
Green, H. J., Lemaire, P., \& Dufau, S. (2007). Eye movement correlates of younger and older adults' strategies for complex addition. Acta Psychologica, 125(3), 257-278. http://doi.org/10. 1016/j.actpsy.2006.08.001

Hodzik, S., \& Lemaire, P. (2011). Inhibition and shifting capacities mediate adults' age-related differences in strategy selection and repertoire. Acta Psychologica, 137(3), 335-344. http://doi.org/10.1016/j.actpsy.2011.04.002

Imbo, I., \& Vandierendonck, A. (2007). The development of strategy use in elementary school children: Working memory and individual differences. Journal of Experimental Child Psychology, 96(4), 284-309. http://doi.org/10.1016/j.jecp.2006.09.001

Koshmider, J. W., \& Ashcraft, M. H. (1991). The development of children's mental multiplication skills. Journal of Experimental Child Psychology, 51(1), 53-89. http://doi.org/10.1016/0022-0965(91)90077-6

LeFevre, J. A., Sadesky, G. S., \& Bisanz, J. (1996). Selection of procedures in mental addition: Reassessing the problem-size effect in adults. Journal of Experimental Psychology, 22, 216-230.

Lemaire, P., Barrett, S.E., Fayol, M., \& Abdi, H. (1994). Automatic activation of addition and multiplication facts in elementary school children. Journal of Experimental Child Psychology, 57, 224-258. http://doi.org/10.1006/jecp.1994.1011

Lemaire, P., \& Siegler, R. S. (1995). Four aspects of strategic change: contributions to children's learning of multiplication. Journal of Experimental Psychology. General, 124(1), 83-97. http://doi.org/10.1037/0096-3445.124.1.83

Lemaire, P., \& Reder, L. (1999). What effects strategy selection in arithmetic? The example of parity and five effects on product verification. Memory \& Cognition, 27(2), 364-382. http://doi.org/10.3758/BF03211420

Lemaire, P., Arnaud, L., \& Lecacheur, M. (2004). Adults' Age-Related Differences in Adaptivity of Strategy Choices: Evidence From Computational Estimation. Psychology and Aging, 19(3), 467-481. http://doi.org/10.1037/0882-7974.19.3.467

Lemaire, P., \& Lecacheur, M. (2011). Age-related changes in children's executive functions and strategy selection: A study in computational estimation. Cognitive development, 26(3), 282-294. http://doi.org/10.1016/j.cogdev.2011.01.002

Mata, R., Schooler, L. J., \& Rieskamp, J. (2007). The aging decision maker: Cognitive aging and the adaptive selection of decision strategies. Psychology and Aging, 22(4), 796-810. http://doi.org/10.1037/0882-7974.22.4.796

Mata, R., von Helversen, B., \& Rieskamp, J. (2010). Learning to choose: Cognitive aging and strategy selection learning in decision making. Psychology and Aging, 25(2), 299-309. http://doi.org/10.1037/a0018923 
Mata, R., \& Nunes, L. (2010). When less is enough: Cognitive aging, information search, and decision quality in consumer choice. Psychology and aging, 25(2), 289-298. http://doi.org/10.1037/a0017927.

McGilly, K., \& Siegler, R. S. (1989). How Children Choose among Serial Recall Strategies. Child Development, 60(1), 172-182. http://doi.org/10.2307/1131083

Otto, A. M. C., Schots, P. A. M., Westerman, J. A. J., \& Webley, P. (2006). Children's use of saving strategies: An experimental approach. Journal of Economic Psychology, 27(1), 57-72. http://doi.org/10.1016/j.joep.2005.06.013

Quaiser-Pohl, C., Rohe, A. M., \& Amberger, T. (2010). The Solution Strategy as an Indicator of the Developmental Stage of Preschool Children's Mental-Rotation Ability. Journal of Individual Differences, 31(2), 95-100. http://doi.org/10.1027/1614-0001/a000017

Reichle, E. D., Carpenter, P. A., \& Just, M. A. (2000). The Neural Bases of Strategy and Skill in Sentence-Picture Verification. Cognitive Psychology, 40(4), 261-295. http://doi.org/10.1006/cogp.2000.0733

Siegler, R. S. (1996). Emerging minds: The process of change in children's thinking. New York: Oxford University Press.

Siegler, R.S. (2007). Cognitive variability. Developmental Science, 10, 104-109. http://doi.org/10.1111/j.1467-7687.2007.00571.x

Stern, Y. (2009). Cognitive reserve. Neuropsychologia, 47(10), 2015-2028. http://doi.org/10. 1016/S1474-4422(12)70191-6

Touron, D. R., \& Hertzog, C. (2004). Distinguishing Age Differences in Knowledge, Strategy Use, and Confidence During Strategic Skill Acquisition. Psychology and Aging, 19(3), 452-466. http://doi.org/10.1037/0882-7974.19.3.452

Touron, D. R., \& Hertzog, C. (2009). Age differences in strategic behavior during a computation-based skill acquisition task. Psychology and Aging, 24(3), 574-585. http://doi.org/10.1037/a0015966

\section{Copyright Disclaimer}

Copyright reserved by the author(s).

This article is an open-access article distributed under the terms and conditions of the Creative Commons Attribution license (http://creativecommons.org/licenses/by/3.0/). 